\title{
Play to test
}

\author{
Carlo Giovannella \\ ScuolalAD \& ISIM_lab \\ University of Rome Tor Vergata \\ via della ricerca scientifica, 1 \\ I - 00133 Rome \\ info@mifav.uniroma2.it
}

\begin{abstract}
To teach and to learn how to test the user behaviour is one of the most delicate aspects of any Interaction Design course because it is usually considered as one of the less creative and more tedious activity of the whole design process. In this paper we show how test activities can be made more pleasant and attractive by focusing on design and use of games. It is also shown how the use of games allows the students to understand the relevance to test multimodal interaction (i.e. combination of visual, motor and emotive channels)
\end{abstract}

\section{Categories and Subject description}

H.5 INFORMATION INTERFACES AND PRESENTATION; H.5.2 [User Interfaces]; K.3 COMPUTER AND EDUCATION; K.3.1 [Computer uses in education]; K.3.2 [Computer and information science education]; K.8 PERSONAL COMPUTING; K.8.0 [General]: Games

\section{General Terms}

Design, Human Factors, Measurements

\section{Keywords}

User behaviour, multimodal interaction, human factors evaluation, games, interaction design, learning.

\section{INTRODUCTION}

During the last decades the test activity has acquired more and more relevance for the design process and nowadays, among the educators, there is a general agreement that it should accompany, although with different modalities, all the phases/layers of the process [1].

In the "real-life", however, the test activity is often confined to the final stage of the process, and often the resources allocated for such an activity are not sufficient to carry on a very accurate investigation. This is the reason why in the past there have been a considerable effort in the development of rapid testing methods, like the discount usability [2], and in the consequent production of a huge amount of guide lines: in a company time is always the king!

This situation derives, to some extend, from a lack of culture about the relevance of a well designed activity of test and this, in turn, may derives from the student perception of such an activity as a quite boring and very time-consuming one. The largest part of the students are convinced that the greatest part of bugs and problems may be discovered and fixed by a rapid self-inspection of the artefact they are developing.

In order to try to "change their mind" we decided to dedicate a special care to the testing activity and, indeed, three years ago we started to assign as practical work for the first term of a two terms course on Interactive and Multimodal Systems the design and realization of a test and, if the case, that of the tools needed to perform it.

Since we are deeply convinced that one needs to understand in its full complexity the multifaceted behaviour of the individuals that develop during their interaction with an artefact [3], and not to restrict to the trivial verification of its functionalities and/or of its usability level, we asked to our students to focus as much as possible on the multidimensionality (visual \& motor, cognitive, emotional, social) of the user behaviour and interaction. We would like to stress, however, that we gave to the students only very general indications not to bias their choices. We tried just to stimulate their creative and divergent thinking about test-design. Only after the completion of such divergent phase we started to interact more strictly to help them to formulate a clearer statement about the goals they intend to achieve during the activity of testdesign\&development.

During the first year of experimentation, we observed that a relevant part of the students reacted more creatively and were more motivated if they were engaged either in testing the people behaviour during games session and/or in designing games to test the people behaviour. Basically this was because the students had the feeling that the outcomes of such an activity could inform the game-design.

In the following we will report on some examples of test activity realized by the students that span on very different aspects of the players' behaviour during their engagement in a "game".

\section{DESIGN AND PLAY TO TEST: SOME EXAMPLES}

Among our student one of the most popular category of game (see for exampe ref. 4) is the action-games After lunch, during the rest time, indeed, they use to play with such games in a multiplayer configuration, interconnecting their computers in a net. Practical reasons for this choice is the possibility to freeze the game situation (by storing it the computer memory) and to resume it later; this possibility, in fact, allows to play only for a limited amount of time. 
Deeper reasons for this choice have probably to do with the particular kind of involvement that is generated by action- games. When used in a multi-player configuration, the action-games often require the assumption of a role and the consequent choice of an avatar for her/his self-representation on the screen and this, making reference to one of the Caillois' categories [5], tends to satisfy the player's need of "mimicry" within a social and interactive context.

More relevant to the content of this paper, however, are two other Caillois' categories: action-games, indeed, satisfy at the same time the "agon" and the "ilinx" conditions and, thus, the expectations of both adults and children. The "agon" is related physically to the battlefield and psychologically to the self-control shown by individuals during a specific game situation. The second one is related to the arousal that derives from the game situation. Sometimes it makes feel like God and can drive people to get involveld in extremely dangerous situations, expecially within protected environments like those offered by the games. Both are strictly related with the emotional level of the interaction and inspired the design of the test that we will discuss in paragraph 2.1. The outcomes of such test, in turns, acted as inspiration for other test activities: in fact one group of students decided to focus on the relationship among visual interaction and motor reaction (see paragraph 2.2), while others on the influence of the playground dimensionality (2D vs. 3D) on the players' behaviour. Actually, this latter is an old interesting problem [6] that has been investigated with regards to the possible benefits that may derive to a learning process from acting in 3D environments. Recently, however, due to the development of huge 3D social space, like Second Life (SL) [7], it became very popular. As far as SL is concerned, recently there are been many claims - actually not fully demonstrated - about the benefits of using 3D environments in learning processes: among them a wider emotional bandwith, a deeper sense of self, a stronger sense of the place, a suspension of disbelief due to the full immersion [8]. All these are very interesting aspects but also very difficult to investigate in a quantitative and objective manner. Thus, our students focused on simpler and more detectable aspects: the influence of the space perception on the fulfilment of very simple games, see paragraph 2.3.

\section{1 "agon", "ilinx" and the interplay between visual and motor behaviour}

Action games, that belong to the "agon" category, certainly involve cognitive and rapid-strategic abilities but involve even more the "ilinx" and the coordination of visual perception and motor reaction. Because of this a group of students decided to focus on a combined detection of visual path (eye-tracking) and biofeedback (body temperature, heartbeat, blood volume pressure - BVP, and tonic constituent of the Galvanic Skin Reflex GSR) and to record the behaviour of 8 subjects during a Quake2 [9] game session 30 seconds long. The subjects have been asked to wear an audio headphone. The eye-tracker calibration was performed twice: before the start of the game session and at its end. The battlefield scene were video recorded for the whole duration of the game session.

Before to start the test session, the students' expectations were to observe visual scan paths covering a consistent portion of the scene and sudden variations of the emotive state during the fire fights. The analysis of the recorded data, however, confirmed only partially their expectations.
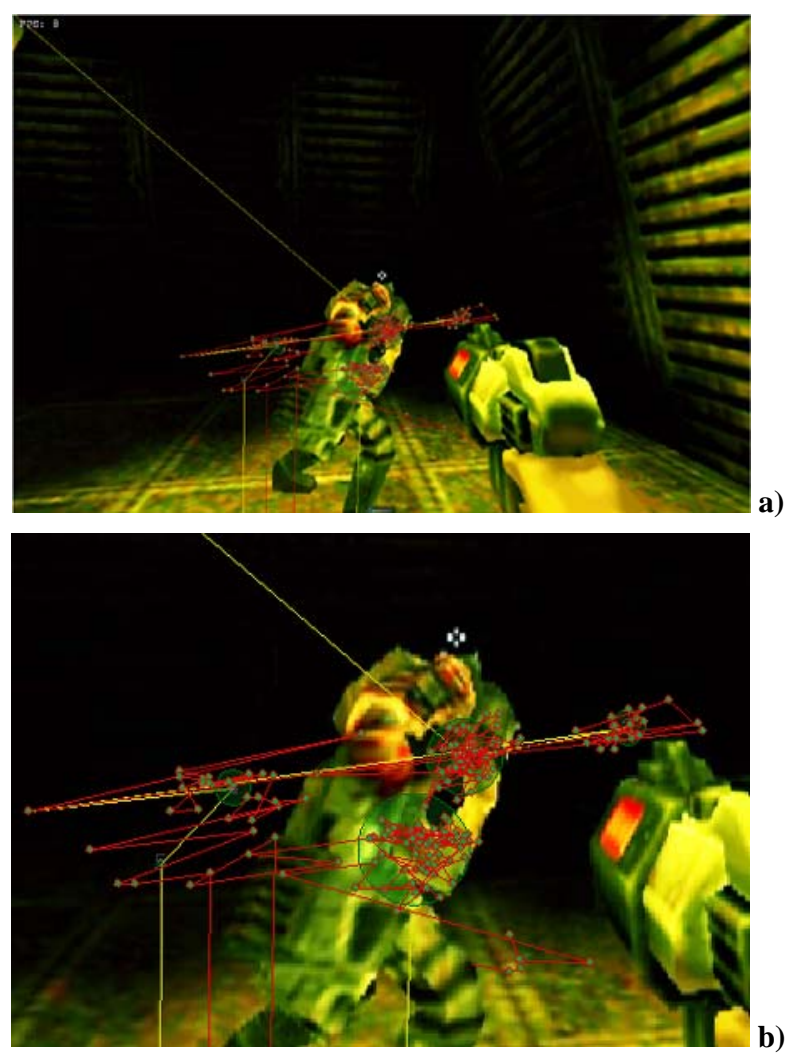

Fig. 1: a) snapshoot from a Quake2 game session; b) zoom in the central portion of the scene to show saccades and fixations.

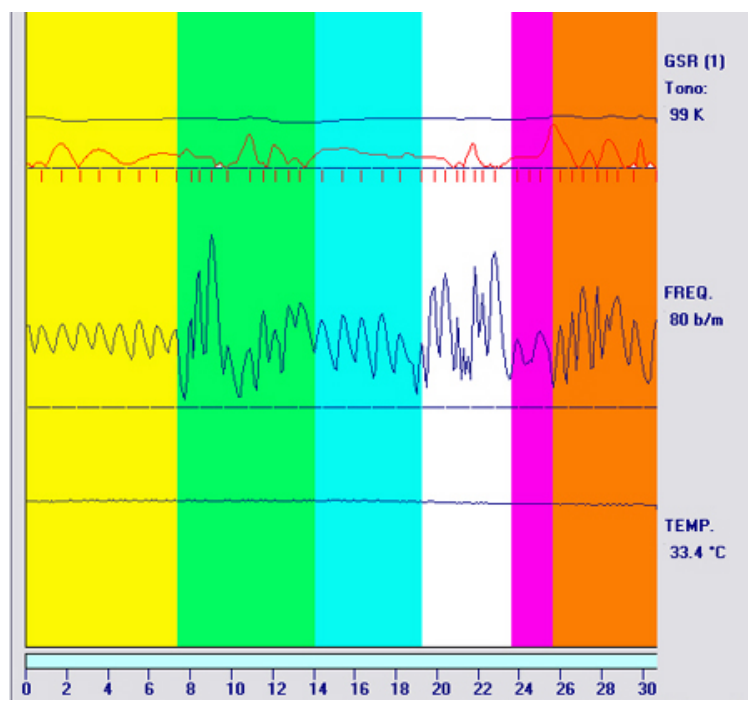

Fig. 2 - An example of biofeedback parameters' recordings

The gaze paths, indeed, showed that the visual attention of all players was focused on the centre of the scene, i.e. where were located both enemies' and weapons' representations, see fig. 1. 
Following these findings, they realized how their initial assumptions were lacking consideration for the 3D nature of the game environment. Indeed the Quake2's scene is a 2D projection of a 3D environment one and in such environment, unlike the 2D ones, the player controls the movements of the camera and almost automatically keeps the point of attention always at the centre of the scene (these observations motivated other students to design new tests to investigate the effect of distractors and of the "alea", but we have not enough room to discuss here this further example of testing activity).

On the other hand the forecasting about the expected variation of the emotional state was confirmed. The recorded data shown that during the whole duration of the test all the players were, more or less, in a state of emotional stress (see the average value of the GSR tonic constituent, 60-120 KOhm, always lower than the value expected for a relaxed state, $200 \mathrm{KOhm})$. Moreover, simultaneously with the fire fights they observed a variation of the heartbeat, see fig. 2, and of the blood volume pressure (BVP) indicating vasoconstrictions and, in turn, an increase of the emotional stress ("ilinx").

\subsection{Ocular exploration, anticipation and the choice of the fastest path in $2 \mathrm{D}$}

Being mainly attracted by the relationship between the visual exploration and the motor reaction, a group of students designed a very simple $2 \mathrm{D}$ game. The playground was designed as a map of a flat (see fig. 3) and the players were asked to go in a time as shortest as possible from the top-right corner of the map to the bottom-left one. Game-design choices included: the adoption of the four keys W-A-S-D standard scheme to move within the 2D space; the representation of the player's position by means of a coloured disk. Before to play the game, the players have been asked to familiarize with the $2 \mathrm{D}$ space by exploring it for 20 seconds, starting from the centre of the playground. This exploration phase have been used to verify the possible influence of coloured objects distributed over the playground on the behaviour of the player.

The students decided to record the position of the avatar and to track the gaze movements of the gamers during both game and exploration sessions.

The students' expectations for the exploration session were that the players would be most attracted by the rooms containing a coloured object (most likely the red one) and that they would have visited all them (for sake of simplicity let's call them red-, blue- and green-room); they also expected to observe a very rapid visual exploration of the whole $2 \mathrm{D}$ environment.

Their expectations for game sessions were: a) a reduction in both the number and the duration of the stops (see black dots in fig. 3a, 3c, 4a, 4c that occur very often before a more or less abrupt change in direction); b) the choice of the principal corridor as the most popular and fastest path to reach the finish; c) an initial fixation of the finish, aimed to take the final decision about the path to follow.

Moreover they also expected, as a very general feature, to observe in both the sessions an ocular anticipation of the avatar trajectory.

5 different players took part in this activity.

The analysis of the recorded data have shown that the players had no specific preference on the room to visit as first target of the exploration, although two of them started from the red-room; 3 over 5 visited all the coloured rooms, 4 over 5 the green-room and 5 over 5 the blue one. The majority of the players performed a very fast ocular exploration of the whole 2D environment, although they spent most of the time in observing the visited rooms. During the exploration phase the players stopped in average 8 times, 1 s/time. The movement of the avatar, as expected, were anticipated by the ocular movement, being the anticipation time included between 0.15 and 0.3 second.

As far as the game phase was concerned the students' forecasting was almost fully fulfilled: the stops were strongly reduced in number (in average no more than two per player); 4 over 5 players decided to go through the vertical corridor it took to them between 7 and 10 second to conclude the game. However the fifth player surprised everyone by choosing an alternative diagonal path and concluding the game in only 5 seconds! Also her/his ocular behaviour was slightly different: in fig. 4 it is shown that the "gaze density" (the time spent by the gaze over a discrete portion of the space) of the fifth player was more homogeneously distributed with respect to those of the other players (see for example fig. 3). It was also interesting to note that: a) the fifth player, unlike the other players, during the explorative phase of the game performed a visual scan of the whole playground, while the motor exploration was confined to a considerably smaller portion of space; b) during the game session, the ocular anticipation of the avatar trajectory appeared to be more relevant in all the players, between 0.2 and $0.5 \mathrm{~s}$, but that of the fifth player was almost the highest, about $0.45 \mathrm{~s}$.

\section{$2.32 \mathrm{D}$ vs. $3 \mathrm{D}$ in practice}

Another group of students decided to push further on the investigation described in the previous paragraph and to design a test to detect differences that may occur when the same game is played either in 3D and in 2D environments.

They designed a game very similar to that described in paragraph 2.2, see fig. 5. The player had to reach one after other four different checking points, represented by coloured cubes (being the last one coincident with the finish of the game). This time, however, the playground was designed in two version: $2 \mathrm{D}$ and $3 D$. The 16 players that took part to the game had to perform two different game sessions using as playground first the $3 \mathrm{D}$ and then the $2 \mathrm{D}$ environment. The players had no possibility to familiarize with the 3D environment but before to start the session they were allowed to visualize a 2D map of the environment to memorize it. They were also allowed to recall the 2D map at any time during the whole game session. The recorded quantities were: position of the avatar, time spent in observing the $2 \mathrm{D}$ maps, times that the player recalled the map during the game session, heartbeat and the blood volume pressure (BVP).

The analysis of the recorded data showed the effectiveness of the map pre-visualization to reach the first checking point (only 4 over 16 players asked to see it again, and only once). On the other hand, most of the players had to check the map again (2 times in average) between the first and the second checking point. Than, during the path to the third checking point, the average number of the map visualizations decreased and increased again during the last stretch of the trail. The students discovered also that a great part of the players experienced a relevant sense of loss, mainly 
due to a design inadvertence: the game-designers, in fact, forgot to show the direction of the camera on the $2 \mathrm{D}$ representation of the space.
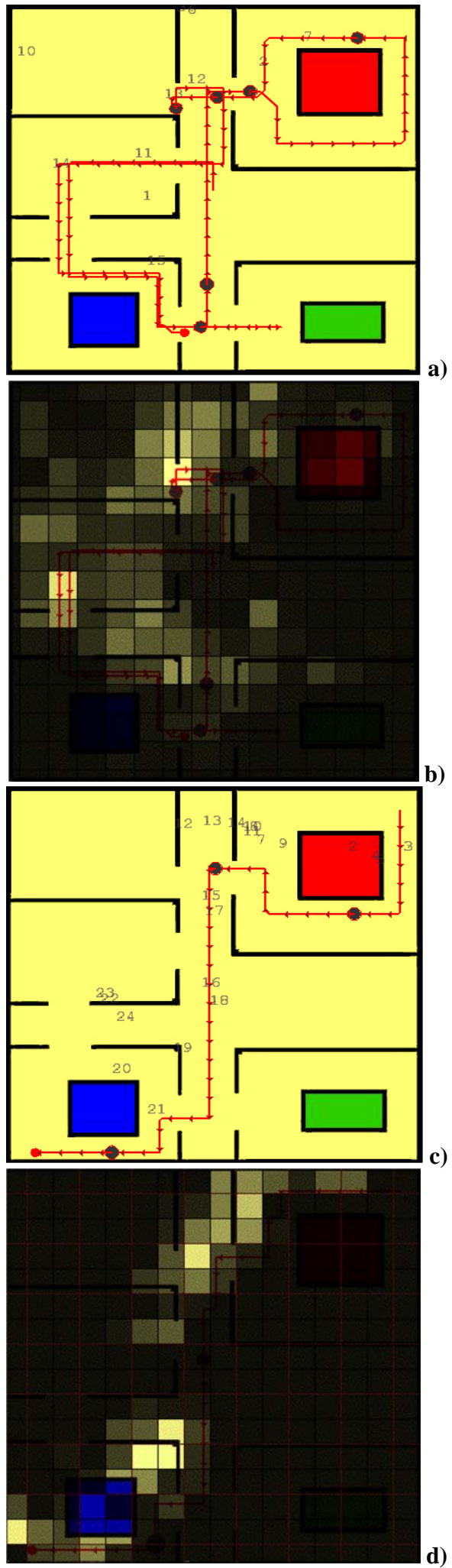

Fig. 3 - a) explorative session (arrowed line: player trajectory); b) visual exploration: density of the gaze; c) same as a) during the game session; same as d) during the game
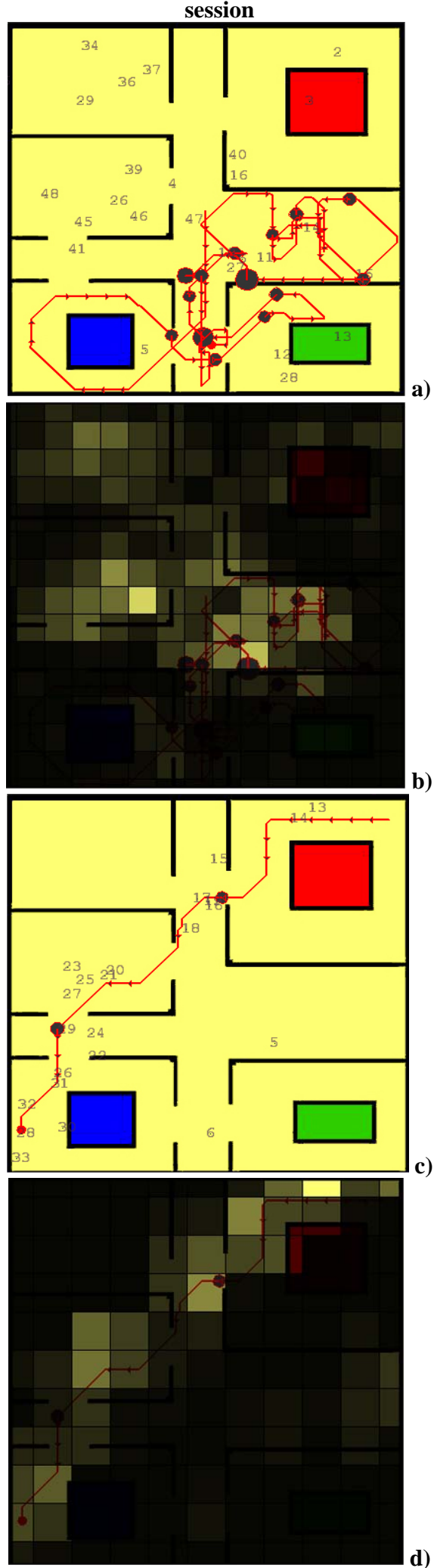
Fig. 4 - Same as fig. 3 for the player that obtained the best performance. Numbers indicate the order of the fixations.

(Both the eye-tracker and the data analysis software are home-made [10])

The above results, together to observations of considerably shorter time intervals needed to finish the game in the 2D playground - in average 55-60\% (measured at each checking point) less with respect to the time needed to accomplish the same task in 3D - arose relevant questions about the uncritical introduction and use of 3D environments.

These observations about the sense of loss confirm the results obtained, some time before, during another test-activity carried on as part of a thesis work devoted to the 3D reconstruction of an anthological exhibition of Pino Pascali that took place in the Rome National Gallery of Art [11]. At that time a 3D scene was used to monitor the behaviour of a visitor faced to the staging of the exhibition. The subjects were divided in two groups, both have been asked to perform a free visit of the exhibition One group was asked to look at the 3D scene on a standard 2D flatscreen, the other to wear a HMD (to experience a more immersive situation).

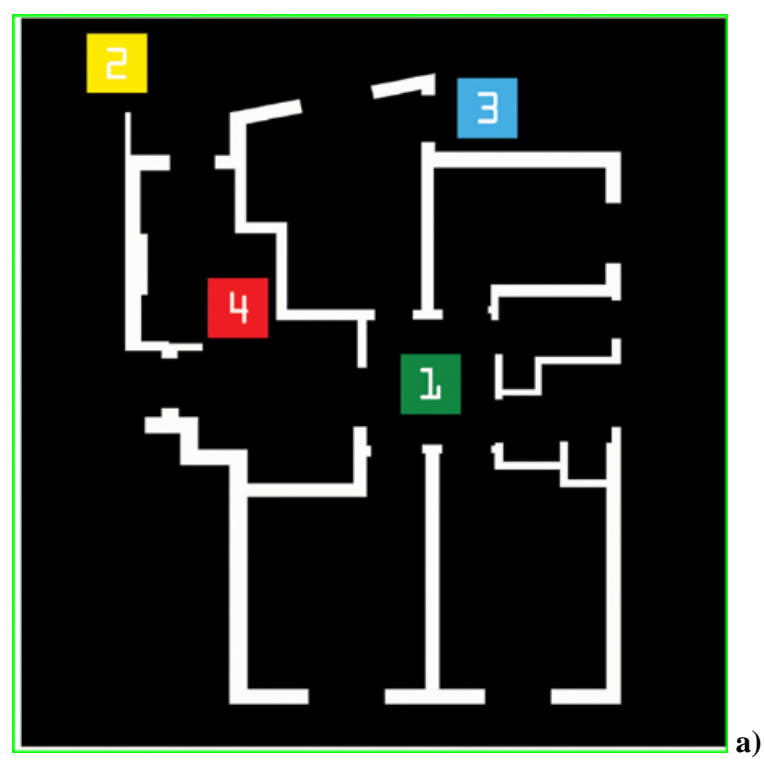

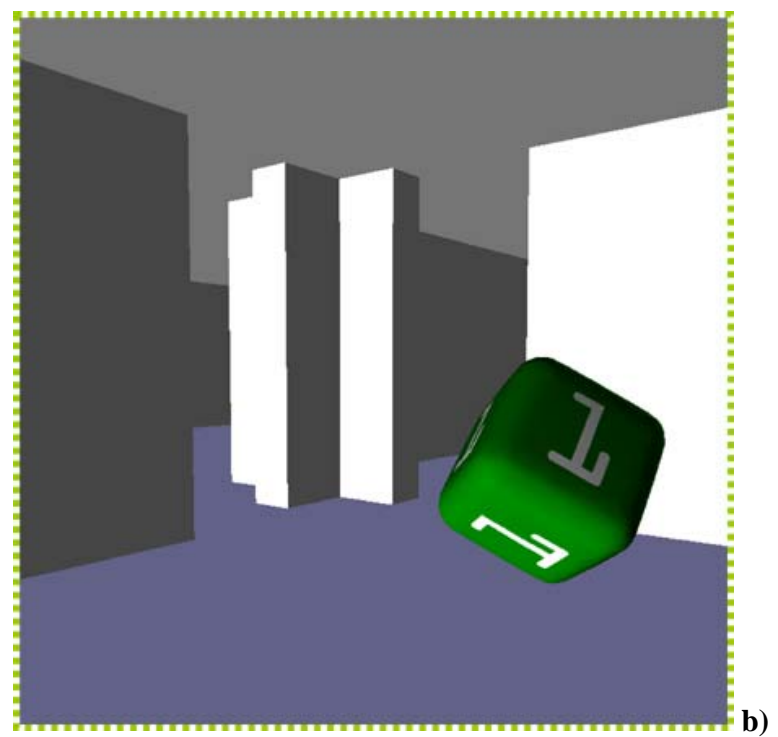

Fig. 5: a) 2D map of the game described in paragraph 2.3; b) a screenshot of the environment used for 3D game sessions
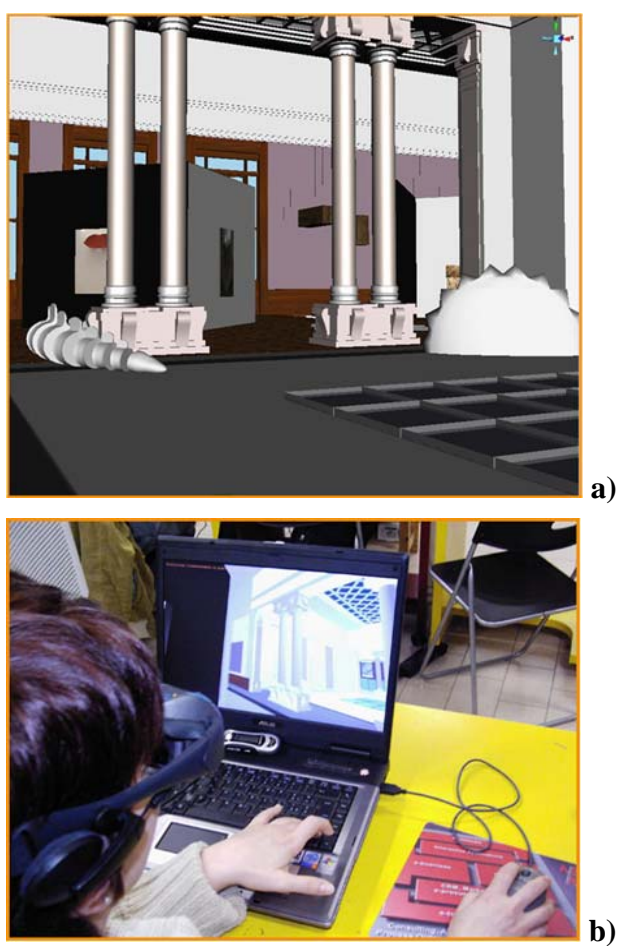

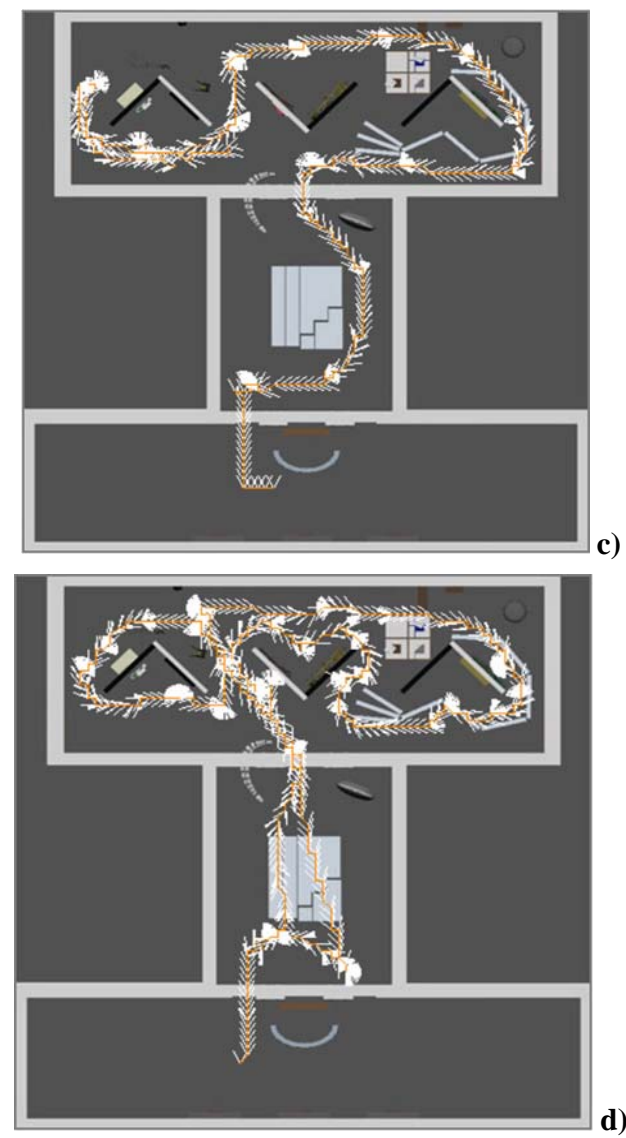

Fig. 6: a) screenshot of the $3 D$ reconstruction of the exhibition; b) immersive experimental setting; c) and d) tracking examples of non-immersive and immersive visits ( the symbol $\mathrm{V}$ indicates the gaze direction)

The visits of the virtual exhibition performed in the immersive set up were slower and, at the same time, the number of large panoramic rotations performed during the exploration doubled reflecting a greater difficulty in orientation. On the other hand the greater difficulty experienced in orienting her/himself had also the positive effect to favour a more complete and, maybe, more accurate visit of the exhibition.

The analysis of the biofeedback recordings allowed the students to investigate and learn a lot about the different gaming styles of the players, however this is a topic that goes beyond the aim of this paper and will be discussed, probably, in an other context. We would like just to mention that the biofeedback recordings showed a general and continuous increase of the arousal during the whole duration of the game that, probably, correlates to the increasing "ilinx" caused by the decreasing distance from the finish. In fact it disappeared immediately after the completion of the game.

\section{LESSON LEARNT AND CONCLUSIONS}

Many other groups of students designed and carried on interesting tests - that involved as an example also the auditory channel - but we do believe that the examples already reported above are enough to demonstrate a possible increase of the students' engagement in activities they usually consider very "boring", when the focus is shifted on gaming. Their involvement in gamedesign (although the examples discussed here dealt with very simple games) and in the use of games to test people behaviour seem to have the following advantages:

- the students learn to observe traces and to identify indicators, to produce testing hypothesis and to design tests (not just to use already designed tests or to test already well known laws), to compare forecasting and results (not just to analyse results);

- they have to reflect on their own game-design choice, not only on the design choices of other designers;

- they understand how dense the interaction can be, not only in the case of very complex applications but even when they are interacting with very simple artefacts and/or services they have designed; and, in turn, they are faced to the full complexity of game-design and, as well, of design for the experiences;

- they feel engaged on forefront subjects (see for example the expectations of the F7P of the EU [12]);

In the past, when we asked the students to elaborate and perform tests on more or less well knows laws - like Fitts law, HickHyman law and so on - their degree of involvement and arousal was, in general, not as high as for the case histories described in this paper. At that time, moreover, we observed also a scarce interest to deepen the tests' outcomes obtained by other groups of students. On the contrary, here, we have seen how new tests and new ideas can be inspired by previous projects and, as well, how new tests may take advantage and be built on top of the previous realizations, as usually happens in every process of innovation (technological, productive, scientific, etc...). In addition, as side effect, we observed that when the interrelationship among different tests, and thus students' groups, becomes stronger also the students' sense of belonging to a learning community increases. This is an aspect to which we are very careful, also by mean of a heavy use of an on-line collaborative learning place like Life [13].

Due to the positive feedback we had, for the future we intend to proceed in this direction; of course we have to refine the methodology and to increase the number of testing facilities ... but the didactic activity, as well known, is always in progress.

In addition we would like to develop an international partnership in order to investigate the issue of multiculturalism.

\section{AKNOWLEDGEMENTS}

I am very grateful to all my students that found the assignments I proposed to them very interesting and challenging, that worked to fulfil their assignments with pleasure (sometime also hard) and that contributed to demonstrate, although "unconsciously", the hypothesis set out in the introduction. It was a great pleasure and an honour to work with all them. A special thanks, of course, goes to Daniele Alaimo, Daniele Alesi, Pietro Di Paolo, Erica Fava, Fabio Listrani, Azzurra Maida, Emanuele Pede, Marco Riommi e Chiara Spadavecchia, whose works have been briefly described in section 2 . All the examples of test activities have been implemented within the course of Interface and Multimodal System I gave (and I am still giving) for the bachelor degree in Media Science and Technology at the University or Rome Tor Vergata. 


\section{REFERENCES}

[1] see for example the papers published in the Proceeedings of the previous HCIEd conferences and the references therein

[2] Nielsen J., Usability Engineering, Morgan Kaufmann Academic Press, San Francisco, 1994

[3] Gaver W.W., Designing for Homo Ludens, in 13 Magazine, 2002

[4] See for example: Lindley C.A ., Game Taxonomies: A High Level Framework for Game Analysis and Design, http://www.gamasutra.com/features/20031003/lindley_01.sht $\mathrm{ml}$ and reference therein

[5] Callois R., Man, Play and Games, First Illinois, 2001 translation from the original Les Jeux et les hommes: le masque et e vertige, Librairie Gallimard, Paris, 1958

[6] Johns C., The Spatial Learning Method: Facilitation of Learning Through The Use of Cognitive Mapping in Virtual Environments, Master Thesis, University of Cape Town, supervised by Blake E., 2002; Serafini L., Rappresentazione e navigazione di mappe concettuali in spazi tridimensionali (in italian), Bachelor Thesis, University of Rome Tor Vergata, supervidsed by Giovannella C., 2003
[7] http://secondlife.com

[8] see for exsmple: Best Practices in Using Virual World for Education at http://www.holymeatballs.org/pdfs/BestPractices.pdf and http://www.simteach.com/wiki/index.php?title=Second_Life_ Education_Wiki;

http://terranova.blogs.com/terra_nova/2007/05/teaching_in_ vr_.html\#more

[9] http://www.quake2.com/

[10] courtesy of prof. C. H. Morimoto and prof. R. Ranvaud USP - Brazil (HW); Giovannella C. (SW) - unpublished

[11] Anthological exhibition of Pino Pascali, GNAM Rome, 15/10/2005 - 27/11/2005, see http://www.exibart.com/notizia.asp?idnotizia=14120

[12] http://cordis.europa.eu/fp7/ict/telearn-digicult/telearnobjectives_en.html

[13] http://life.mifav.uniroma2.it 